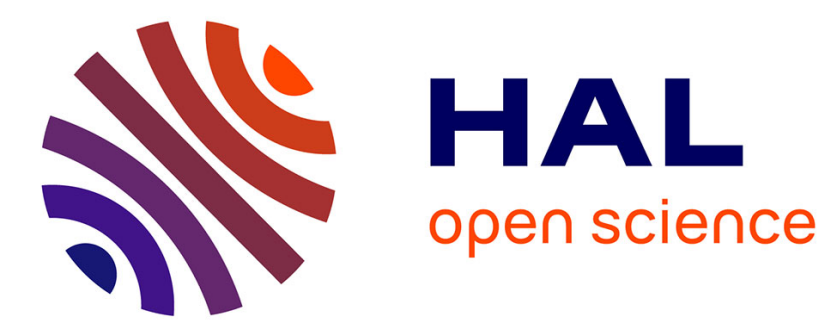

\title{
Phonon limited anisotropic quantum transport in phosphorene field effect transistors
}

Madhuchhanda Brahma, Arnab Kabiraj, Marc Bescond, Santanu Mahapatra

\section{To cite this version:}

Madhuchhanda Brahma, Arnab Kabiraj, Marc Bescond, Santanu Mahapatra. Phonon limited anisotropic quantum transport in phosphorene field effect transistors. Journal of Applied Physics, 2019, 126 (11), pp.114502. 10.1063/1.5109057 . hal-02331920

\section{HAL Id: hal-02331920 \\ https://hal.science/hal-02331920}

Submitted on 20 Nov 2020

HAL is a multi-disciplinary open access archive for the deposit and dissemination of scientific research documents, whether they are published or not. The documents may come from teaching and research institutions in France or abroad, or from public or private research centers.
L'archive ouverte pluridisciplinaire HAL, est destinée au dépôt et à la diffusion de documents scientifiques de niveau recherche, publiés ou non, émanant des établissements d'enseignement et de recherche français ou étrangers, des laboratoires publics ou privés. 


\title{
Modeling of Anisotropic Dissipative Quantum Transport in Phosphorene Field Effect Transistors
}

\author{
Madhuchhanda Brahma,,${ }^{1,2, *}$ Arnab Kabiraj, ${ }^{2}$ Marc Bescond, ${ }^{3}$ and Santanu Mahapatra ${ }^{2}$ \\ ${ }^{1}$ Centre for Nanoscience and Engineering, Indian Institute of Science, Bangalore-560012, India \\ ${ }^{2}$ Nanoscale Device Research Laboratory, Department of Electronic Systems Engineering, \\ Indian Institute of Science, Bangalore-560012, India \\ ${ }^{3}$ LIMMS, CNRS-UMI 2820, Institute of Industrial Science, University of Tokyo, 153-8505 Tokyo, Japan
}

\begin{abstract}
Dissipative transport in $20 \mathrm{~nm}$ long phosphorene metal oxide semiconductor field effect transistor (MOSFET) is studied in armchair (AC) and zigzag (ZZ) directions. In a multiscale approach, the unit cell of phosphorene is first relaxed and bandstructure is calculated using hybrid density functional theory (DFT). The transport equations are then solved quantum mechanically under the nonequilibrium Green's function (NEGF) formalism using DFT-calibrated two band $\mathbf{k} \cdot \mathbf{p}$ hamiltonian. The treatment of electron phonon scattering is done under the self consistent Born approximation (SCBA) in conjunction with deformation potential theory. It is found that, optical phonon modes are largely responsible for degradation of ON-current apart from $\mathrm{p}$ channel AC MOSFET where acoustic phonon modes play a stronger role. It is further observed that electron-phonon scattering is more pronounced in $\mathrm{ZZ}$ direction whereas the diffusive ON-current of p-MOSFET in a given direction is higher than n-MOSFET. Further study on the complex bandstructure of phosphorene reveals band wrapping within bandgap region in AC direction and multiple crossings in ZZ direction. This signifies strong phonon assisted tunneling (PAT) in ZZ direction in comparison to AC direction. For completeness, drain current in AC tunnel field effect transistor (TFET) is calculated and electron-phonon scattering is observed only in the near vicinity of the OFF current.
\end{abstract}

PACS numbers: 73.63.-b,71.15.Mb,85.35.-p,71.38.-k

\section{INTRODUCTION}

Atomically thin two dimensional (2D) layered materials has ushered in a new era in the field of semiconductor device and technology. These materials and their potential applications for transistor technologies are being studied both experimentally and theoretically. Most of these $2 \mathrm{D}$ materials such as $\mathrm{hBN}$, phosphorene, transition metal di-chalcogenides (TMD), like $\mathrm{MoS}_{2}, \mathrm{WS}_{2}, \mathrm{MoSe}_{2}$, $\mathrm{WSe}_{2}, \mathrm{MoTe}_{2}[1-6]$ are being extensively explored as an alternative channel material for MOSFET, in order to meet the Moore's law projections.

Among these materials, phosphorene, a single atomic layer of black phosphorus with a puckered honeycomb lattice structure $[4,5]$, has warrented special interest for its highly anisotropic transport properties. It has also resulted in successful fabrication of transistors [4, 5, 711]. Theoretical assesments [11-15] reveal that phosphorene MOSFETs outperform the TMD based MOSFETs in terms of ON-current requirements and subthreshold swing characteristics. The noteworthy performance of phosphorene MOSFETs is attributed to its very low effective mass along the armchair direction.

Most of the theoretical studies on phosphorene FETs have been limited to ballistic transport. Only few recent articles have reported the performance of phosphorene MOSFET under electron-phonon scattering $[14,15]$. However, the channel length considered in those studies

*madhuchhanda@iisc.ac.in is limited to $10 \mathrm{~nm}$, which is close to the ballistic limit. Current spectrum profiles in Ref. [14] suggest that the possible detrimental effect of phonon scattering on the performance of MOSFET fades out at such shorter channel lengths. In this work, we then calculate the dissipative transport in phosphorene MOSFET with a longer channel length $(20 \mathrm{~nm})$. In addition, we also inspect the effect of electron-phonon scattering on the performance of tunnel field effect transistor (TFET), which has attracted attention for low-power applications. In contrast to previous studies, we investigate the individual effect of different phonon modes on the drain current of those devices.

Non-equilibrium Green's function (NEGF) equations expressed in the self-consistent Born approximation (SCBA) are used to calculate dissipative transport in $\mathrm{n}$ and $\mathrm{p}$ type phosphorene MOSFETs in both armchair (AC) and zigzag (ZZ) directions as well as p-i-n tunnel FET. The paper is organized as follows. In Section II the simulation methodology is described followed by section III where the effect of different electron-phonon interaction on the device transfer characteristics are pointed out. We finally draw our concluding remarks in Section IV. The present work is an extended version of our recent presentation [16], which was based on single band effective mass hamiltonian and limited to n-channel MOSFETs. 


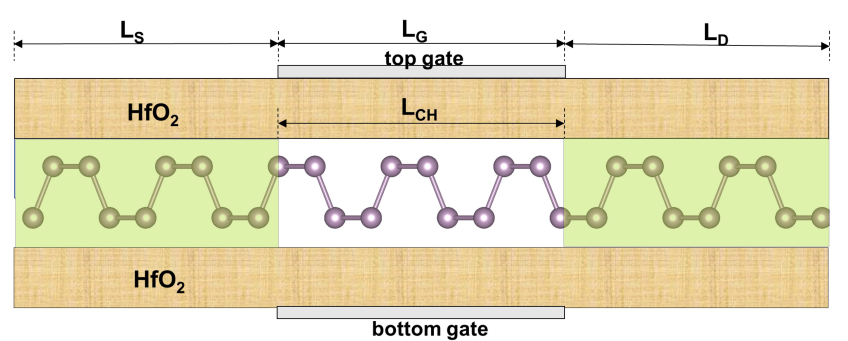

(a)

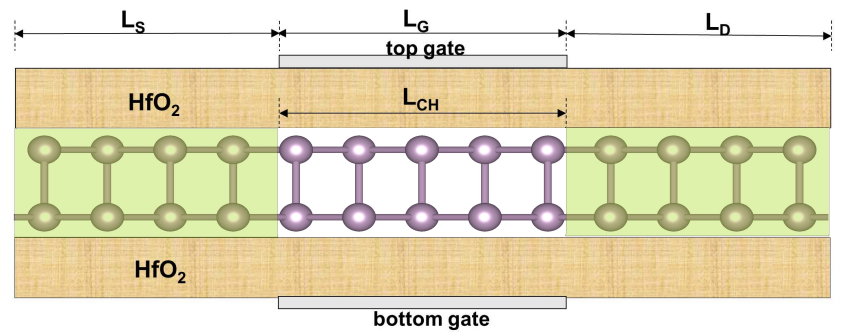

(b)

FIG. 1. Cross-section of double-gate phosphorene MOSFET with transport direction as (a) armchair and (b) zigzag. $L_{\mathrm{CH}}$ denotes the channel length, which is set equal to the gate length $L_{\mathrm{G}}$. The channel is undoped, while the source and drain extensions (shaded) are uniformly doped in MOSFET, and $\mathrm{p}$ and $\mathrm{n}$ type respectively in TFET

\section{METHODOLOGY}

A schematic representation of the device cross-section under consideration is shown in FIG. 1. The effective oxide thickness (EOT) is chosen as $0.41 \mathrm{~nm}$ and supply bias $V_{\mathrm{D}}=0.64 \mathrm{~V}$, according to the ITRS 2013 specifications [17]. The length $L_{\mathrm{CH}}$ of the undoped channel is taken equal to the gate length $L_{\mathrm{G}}$ which is $20 \mathrm{~nm}$. In case of MOSFET, $20 \mathrm{~nm}$ long source and drain extensions are uniformly doped at $3 \times 10^{13} / \mathrm{cm}^{2}$ whereas in TFET they are doped at $2 \times 10^{13} / \mathrm{cm}^{2}$ (p type) and $1 \times 10^{13} / \mathrm{cm}^{2}$ (n type) respectively. Thickness of the phosphorene slab as extracted from the atomic configuration used in density functional theory (DFT) calculations turns to be $0.6 \mathrm{~nm}$. The OFF-state current $\left(I_{\mathrm{OFF}}\right)$ at zero gate voltage $\left(V_{\mathrm{G}}\right)$ is set at $100 \mathrm{nA} / \mu \mathrm{m}$ in MOSFET and at $10 \mathrm{nA} / \mu \mathrm{m}$ in TFET by choosing an appropriate gate work function.

DFT calculations are performed to evaluate the electronic band structure, carrier effective mass and band gap values. The calculations are carried out using generalized gradient approximation (GGA) as implemented in the code VASP [18-20] with PAW [21] method using the Perdew-Burke-Ernzenhof (PBE) [22] exchangecorrelation and the Heyd-Scuseria-Ernzerhof (HSE)[23] hybrid functional. The wavefunctions obtained from the PBE calculations are only used as a starting point in the HSE calculations. All the structural as well as electronic properties except the complex bandstruc- ture (CBS) and phonon spectrum are determined from the HSE calculations. The lattice parameters used for electronic structure calculations are $\mathrm{a}=4.57(4.62) \AA$ and $\mathrm{b}=3.27(3.29) \AA$ for HSE(PBE) functional based DFT. These values are in agreement with other reported results $[24,25] .3 \mathrm{~s}^{2} 3 \mathrm{p}^{3}$ electrons of Phosphorus are treated as valence electrons and expanded in plane wave basis set. A cutoff energy of $400 \mathrm{eV}$ is used and a $\Gamma$-centered $15 \times 13 \times 1$ (in $\mathrm{X}, \mathrm{Y}$ and $\mathrm{Z}$ directions) $\mathrm{k}$ mesh is found to be suitable to sample the Brillouin zone for structural relaxations and a denser $\mathrm{k}$ mesh of $33 \times 29 \times 1$ is used for static calculations. Electronic convergence is achieved when the difference in energy of successive electronic steps becomes less than $10^{-6} \mathrm{eV}$, whereas the structural geometry is optimized until the maximum force on every atom falls below $10^{-6} \mathrm{eV} / \AA$. A large vacuum space of more than $20 \AA$ in the direction of $\mathrm{Z}$ is applied to avoid any interaction between successive layers. Phonon calculations are done using the code Phonopy [26] which uses the atomic forces calculated from VASP based on density functional perturbation theory (DFPT) applied to a $2 \times 2$ phosphorene unit cell using a $27 \times 25 \times 1 \mathrm{k}$-mesh. For generating CBS, DFT calculations are performed using Atomistix Tool Kit (ATK) [27]. The accuracy of the calculations largely depends on the selection of norm-conserving pseudopotentials and numerical linear combination of atomic orbitals (LCAO) basis sets in this case. GGA is employed as the exchange correlation in conjunction with the PBE functional. The 'SG15' norm-conserving pseudopotentials with 'medium' basis sets have been used. The optimized 'SG15' provide smooth pseudopotentials with multiple projectors and non-linear core corrections $[28,29]$. A k-mesh with the density of $6 \AA$ along every lattice vectors is chosen and a cutoff energy of 60 Hartree is employed. In order to obtain the CBS along AC and $\mathrm{ZZ}$ directions, the phosphorene surface is cleaved along [100] and [010]. The value of bandgap obtained by using PBE functional is $0.9 \mathrm{eV}$ and from HSE is $1.61 \mathrm{eV}$. The classical local or semi-local DFT is known to severely underestimate the band gap of a material severely and can derive inaccurate structural parameters as well. The HSE hybrid functional includes $25 \%$ of Fock non-local exact exchange energy for short-range interactions which overcomes these limitations of local and semi-local DFT, and provides a good estimation of electronic as well as structural parameters for most systems. However, calculation of the Fock exchange increases the computational cost manifold and is thus not suitable for complex band structure calculation. On the other hand, HSE functional based DFT data are used for transport calculations since it is more rigorous.

Phonon spectrum of phosphorene generated from VASP is illustrated in FIG. 2. The different modes are numbered according to Ref. [30]. Phonon modes 2 and 3 stand for the acoustic branches whereas 7, 9 and 11 denote the optical branches. The corresponding deformation potential values and the frequencies of the optical phonon branches are represented in TABLE. I. Among 


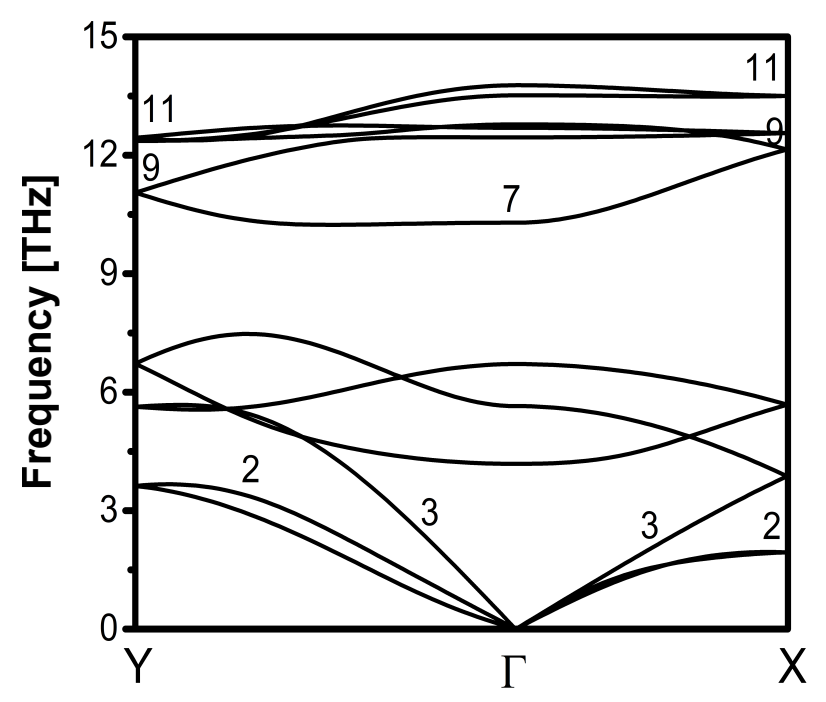

FIG. 2. Phonon dispersion of phosphorene obtained from VASP. The different phonon branches included in our simulation are numbered. Branches 2 and 3 are acoustic phonons while the remaining marked branches stand for different optical modes [30].

the two acoustic modes, 2 is considered for transport across $\mathrm{AC}$ direction whereas 3 for $\mathrm{ZZ}$ direction. This is due to the dominance of the respective modes along $\mathrm{AC}$ or $\mathrm{ZZ}$ direction as demonstrated in the contour plot of electron-phonon coupling elements in Ref. [30]. Zero order optical phonon modes are considered in transport calculations. The effect of first order optical modes on the total scattering rates is insignificant [30] with respect to the zero order modes and thus not considered in this study [31].

The electronic band dispersion is modeled using a twoband $\mathbf{k} \cdot \mathbf{p}$ hamiltonian [25].

$$
H(\mathbf{k}) \equiv\left[\begin{array}{cc}
E_{c}+\eta_{c} k_{x}^{2}+\nu_{c} k_{y}^{2} & \gamma_{1} k_{x}+\beta k_{y}^{2} \\
\gamma_{1} k_{x}+\beta k_{y}^{2} & E_{v}+\eta_{v} k_{x}^{2}+\nu_{v} k_{y}^{2}
\end{array}\right]
$$

where $\mathbf{k}=\left(k_{x}, k_{y}\right)$ is the in-plane wave vector, $\eta_{c(v)}$, $\nu_{c(v)}$ and $\gamma_{1}$ are the fitting parameters, $E_{c}$ and $E_{v}$ are the conduction band minimum and valence band maximum. The eigen values of the hamiltonian are calibrated against the DFT generated dispersion by choosing appropriate values of the fitting parameters, as shown in TABLE. II. It is found (see FIG. 3)that band dispersion obtained from single band effective mas hamiltonian matches DFT data in a small energy window, whereas the band dispersion from two-band hamiltonian shows excellent match over a broad energy spectrum suitable for transport calculations in both MOSFETs and TFETs.

For transport calculation, the NEGF equations are solved self-consistently with the Poisson's equation. Assumption of translational invariance in the $y(x)$ direction of the device (according to the direction of transport i.e.

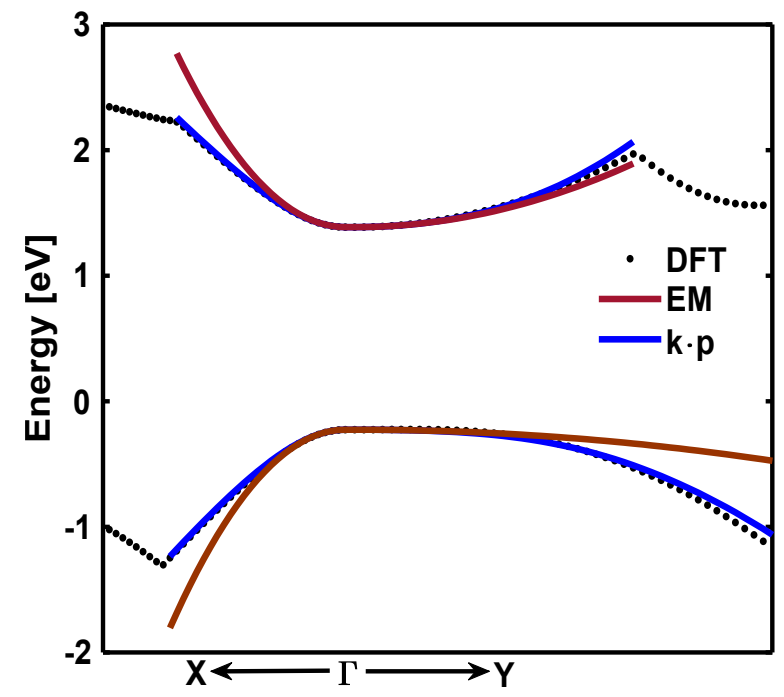

FIG. 3. Fitting of the bandstructure from model hamiltonian with DFT generated band dispersion. The eigenvalues from two band hamiltonian shows excellent match over an energy interval of $0.5 \mathrm{eV}$ whereas the single band effective mass (EM) hamiltonian deviates from the actual dispersion.

$\mathrm{AC}$ or $\mathrm{ZZ}$ ), enables to express the hamiltonian and the Green's functions in terms of the in-plane transversal wave vector $k_{y(x)}$. Born-von Karman periodic boundary conditions are imposed in a system with finite length $\mathrm{L}_{y(x)}=80 \mathrm{~nm}$ by including 20 uniformly spaced transversal wave vectors $k_{y(x), n}=2 \pi n / \mathrm{L}_{y(x)}$, where $n$ denotes the index of the transverse mode. For each wave vector $k_{y(x)}$, the retarded Green's function (expressed in the matrix notation) is calculated at each energy $E$ as [32-34]

$$
\begin{array}{r}
G^{\mathrm{r}}\left(k_{y(x)}, E\right)=\left[(E-U) I-H\left(k_{y(x)}\right)-\Sigma_{\mathrm{S}}^{\mathrm{r}}-\Sigma_{\mathrm{D}}^{\mathrm{r}}\right. \\
\left.-\Sigma_{\mathrm{ph}(\mathrm{ac})}^{\mathrm{r}}-\Sigma_{\mathrm{ph}(\mathrm{op})}^{\mathrm{r}}\right]^{-1}
\end{array}
$$

where $U$ is the electrostatic potential in the device, $I$ is the identity matrix, $H\left(k_{y(x)}\right)$ is the hamiltonian matrix discretized along the transport direction $x(y), \Sigma_{\mathrm{S}}^{\mathrm{r}}$ and $\Sigma_{\mathrm{D}}^{\mathrm{r}}$ are retarded self-energy matrices associated to the source/drain contacts [32], $\Sigma_{\mathrm{ph}(\mathrm{ac})}^{\mathrm{r}}$ and $\Sigma_{\mathrm{ph}(\mathrm{op})}^{\mathrm{r}}$ are the retarded self-energy matrices due to interaction between carriers and acoustic (ac) and optical phonons (op) respectively. From the retarded Green's function $G^{\mathrm{r}}\left(k_{y(x)}, E\right)$ in Eq. (1), one can calculate the lesser (i) and greater (i) Green's functions, $G^{\lessgtr}\left(k_{y(x)}, E\right)$ as follows:

$$
\begin{aligned}
G^{\lessgtr}\left(k_{y(x)}, E\right)= & G^{\mathrm{r}}\left(k_{y(x)}, E\right)\left[\Sigma_{\mathrm{S}}^{\lessgtr}\left(k_{y(x)}, E\right)\right. \\
+\Sigma_{\mathrm{D}}^{\lessgtr} & \left(k_{y(x)}, E\right)+\Sigma_{\mathrm{ph}(\mathrm{ac})}^{\lessgtr}\left(k_{y(x)}, E\right) \\
& \left.+\Sigma_{\mathrm{ph}(\mathrm{op})}^{\lessgtr}\left(k_{y(x)}, E\right)\right] G^{\mathrm{r} \dagger}\left(k_{y(x)}, E\right)
\end{aligned}
$$

where $\Sigma_{S / D}^{\lessgtr}$ are the lesser $(i)$ and greater $(i)$ self-energies 
TABLE I. Values of deformation potentials $\mathrm{D}$ (in unit $10^{8} \mathrm{eV} / \mathrm{cm}$ for optical and in $\mathrm{eV}$ for acoustic) and phonon energies $\hbar \omega$ (in $\mathrm{meV}$ ) for various numbered branches in the phonon spectrum [30] for both electrons and holes.

\begin{tabular}{|c|c|c|c|c|c|c|c|c|c|c|}
\hline \multirow{3}{*}{ Parameter } & \multicolumn{4}{|c|}{ Acoustic } & \multicolumn{6}{|c|}{ Optical } \\
\hline & \multicolumn{2}{|c|}{$2(\mathrm{AC})$} & \multicolumn{2}{|c|}{$3(\mathrm{ZZ})$} & \multicolumn{2}{|c|}{7} & \multicolumn{2}{|c|}{9} & \multicolumn{2}{|c|}{11} \\
\hline & elec. & hole & elec. & hole & elec. & hole & elec. & hole & elec. & hole \\
\hline $\mathrm{D}$ & 2.74 & 4.21 & 8.31 & 1.83 & 8.07 & 1.22 & 6.60 & 3.81 & 6.60 & 3.81 \\
\hline$\hbar \omega$ & - & - & - & - & \multicolumn{2}{|c|}{42.1} & \multicolumn{2}{|c|}{51.9} & \multicolumn{2}{|c|}{55.0} \\
\hline
\end{tabular}

TABLE II. Values for $\mathbf{k} \cdot \mathbf{p}$ hamiltonian parameters used in transport calculation and effective mass for electrons (e) and holes (h).

\begin{tabular}{|c|c|c|c|c|c|c|c|}
\hline \multicolumn{6}{|c|}{ Calibrated Parameters for k·p hamiltonian } & \multicolumn{2}{c|}{ effective mass } \\
\hline$\eta_{c}\left(\mathrm{eV} \AA^{2}\right)$ & $\eta_{v}\left(\mathrm{eV}^{2}\right)$ & $\nu_{c}\left(\mathrm{eV} \AA^{2}\right)$ & $\nu_{v}\left(\mathrm{eV} \AA^{2}\right)$ & $\gamma_{1}(\mathrm{eV} \AA)$ & $\beta\left(\mathrm{eV} \AA^{2}\right)$ & $m_{x}\left(m_{0}\right)$ & $m_{y}\left(m_{0}\right)$ \\
\hline 1.34 & 2.61 & 2.91 & 0.63 & 5.82 & 3.72 & $0.17(\mathrm{e}), 0.16(\mathrm{~h})$ & $1.29(\mathrm{e}), 5.96(\mathrm{~h})$ \\
\hline
\end{tabular}

in the source $(\mathrm{S})$ and drain $(\mathrm{D})$ contacts, calculated as

$$
\begin{aligned}
& \Sigma_{\mathrm{S} / \mathrm{D}}^{<} \equiv\left(\Sigma_{\mathrm{S} / \mathrm{D}}^{\mathrm{r}}-\Sigma_{\mathrm{S} / \mathrm{D}^{\dagger}}\right) \cdot \mathrm{f}_{\mathrm{S} / \mathrm{D}} \\
& \Sigma_{\mathrm{S} / \mathrm{D}}^{>} \equiv\left(\Sigma_{\mathrm{S} / \mathrm{D}}^{\mathrm{r}}-\Sigma_{\mathrm{S} / \mathrm{D}}^{\mathrm{r}}{ }^{\dagger}\right) \cdot\left(1-\mathrm{f}_{\mathrm{S} / \mathrm{D}}\right)
\end{aligned}
$$

Here $\dagger$ denotes the conjugate transpose and $\mathrm{f}_{\mathrm{S} / \mathrm{D}}$ represents the Fermi-Dirac distribution function in the source/drain. $\Sigma_{\mathrm{ph}(\mathrm{ac})}^{\lessgtr}$ and $\Sigma_{\mathrm{ph}(\mathrm{op})}^{\lessgtr}$ in Eq. (2) stand for the acoustic and optical scattering self energies respectively.

The dissipative transport is treated within the selfconsistent Born approximation (SCBA). Under such approximation, the lesser $(i)$ and greater $(i)$ self-energies for acoustic and optical phonon interaction are $[33,34]$ given as follows. Only the diagonal elements of Green's function are considered based on the assumption that carrier-phonon interactions are local.

$$
\Sigma_{\mathrm{ph}(\mathrm{ac})}^{\lessgtr}(j, j, E)=\sum_{k_{x(y)}} \sum_{v} \mathrm{D}_{\mathrm{v}(\mathrm{ac})} G^{\lessgtr}\left(j, j, k_{x(y)}, E\right)
$$

$$
\begin{gathered}
\sum_{\mathrm{ph}(\mathrm{op})}^{\lessgtr}(j, j, E)=\sum_{k_{x(y)}} \sum_{v} \mathrm{D}_{\mathrm{v}(\mathrm{op})}\left(\mathrm{N}_{\mathrm{B}}+\frac{1}{2} \pm \frac{1}{2}\right) \\
G^{\lessgtr}\left(j, j, k_{x(y)}, E \pm \hbar \omega_{\mathrm{ph}}\right)
\end{gathered}
$$

where $j$ is the position index of the lattice grid point, $v$ is the phonon mode, $\omega_{\mathrm{ph}}$ is the phonon frequency, $\mathrm{N}_{\mathrm{B}}=\left[\exp \left(\hbar \omega_{\mathrm{ph}} / \mathrm{k}_{\mathrm{B}} \mathrm{T}\right)-1\right]^{-1}$ is the Bose factor for phonon occupancy, $\mathrm{D}_{\mathrm{v}}(\mathrm{ac} / \mathrm{op})$ denotes the square of the electron phonon matrix element from the deformation potential theory. The terms $\mathrm{D}_{\mathrm{v}(\mathrm{ac} / \mathrm{op})}$ in Eqs. (3) and (4) are determined as

$$
\begin{aligned}
& \mathrm{D}_{\mathrm{v}(\mathrm{ac})}=\Xi_{\mathrm{ac}}^{2} \mathrm{k}_{\mathrm{B}} \mathrm{T} / \rho \mathrm{Vv}_{\mathrm{p}}^{2} \\
& \mathrm{D}_{\mathrm{v}(\mathrm{op})}=\Xi_{\mathrm{op}}^{2} \hbar^{2} / 2 \rho \mathrm{VE}_{\mathrm{ph}}
\end{aligned}
$$

where $\Xi_{\mathrm{ac}}$ and $\Xi_{\mathrm{op}}$ denote the acoustic and optical deformation potential constants, $\rho$ is the volumetric mass density of the material, $\mathrm{v}_{\mathrm{p}}$ is the sound velocity and $\mathrm{E}_{\mathrm{ph}}$ denotes the optical phonon energy. The retarded selfenergies for both acoustic (ac) and optical (op) phonon scattering is calculated from the lesser and greater self energies as [34, 35]

$$
\Sigma_{\mathrm{ph}(\mathrm{ac})}^{\mathrm{r}}(j, j, E)=\sum_{k_{x(y)}} \sum_{v} \mathrm{D}_{\mathrm{v}(\mathrm{ac})} G^{\mathrm{r}}\left(j, j, k_{x(y)}, E\right)
$$



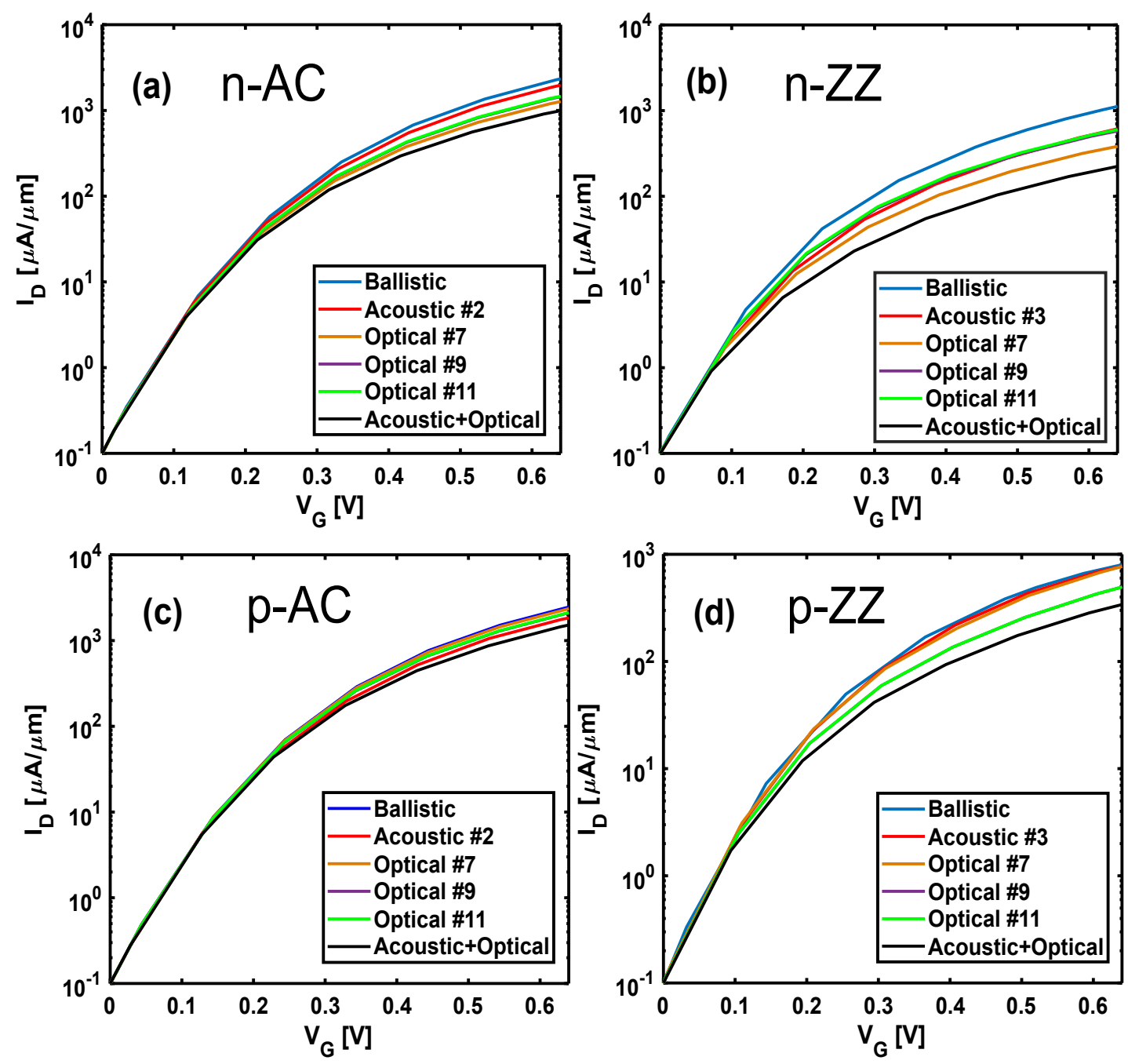

FIG. 4. Simulated transfer characteristics of phosphorene n-MOSFETs along (a) armchair(AC) and (b) zigzag(ZZ) directions and p-MOSFETs along (c) AC and (d) ZZ directions. In the ballistic regime, Poisson's equation does not converge anymore above $V_{\mathrm{G}}=0.5 \mathrm{~V}$ along zigzag directions due to oscillations in the source region [36]. Ballistic current values are then obtained by extrapolation for $V_{\mathrm{G}}$ above $0.5 \mathrm{~V}$.

tions, as it mainly contributes to an energy renormalization [? ? ? ]. The interdependence between the scattering self-energies and the Green's functions demands for an iterative solution of Eqs. (1)-(6) until self-consistency is reached by preserving the charge and current conservation laws [36]. In order to accelerate such self-consistent loop, the recursive Green's function algorithm is used $[33,37]$. Once the self-consistent Born approximation loop is converged, the electron and hole densities are determined from the Green's functions $G^{\lessgtr}$ as

$$
\begin{aligned}
& n(j, j) \equiv \frac{1}{2 \pi} \sum_{k_{y(x)}} \int_{-\infty}^{+\infty} G^{<}\left(j, j, k_{y(x)}, E\right) d E \\
& p(j, j) \equiv \frac{1}{2 \pi} \sum_{k_{y(x)}} \int_{-\infty}^{+\infty} G^{>}\left(j, j, k_{y(x)}, E\right) d E
\end{aligned}
$$

The current density from position $j$ to $j+1$ along the $x(y)$ direction is calculated from the off-diagonal elements

$$
\begin{aligned}
& (j, j+1) \text { of } G^{\lessgtr}\left(i, j, k_{y(x)}, E\right) \text { as } \\
& J \lessgtr(i, i+1) \equiv \sum_{k_{y(x)}} \frac{2 \mathrm{q}}{\hbar \mathrm{S}} \int_{-\infty}^{+\infty} \frac{d E}{2 \pi}\left[H(j, j+1) G^{\lessgtr}((j+1, j),\right. \\
& \left.\left.k_{y(x)}, E\right)-H(j+1, j) G^{\lessgtr}\left((j, j+1), k_{y(x)}, E\right)\right]
\end{aligned}
$$

where $H(j, j+1)$ corresponds to the nearest neighbour hopping terms in the discretized hamiltonian. Finite volume method is used to discretize the 2D Poisson's equation across the device cross-section shown in FIG. 1. Dirichlet boundary conditions are enforced at the metal gate electrodes whereas Neumann boundary conditions are used on the rest of the edges to satisfy charge neutrality. 

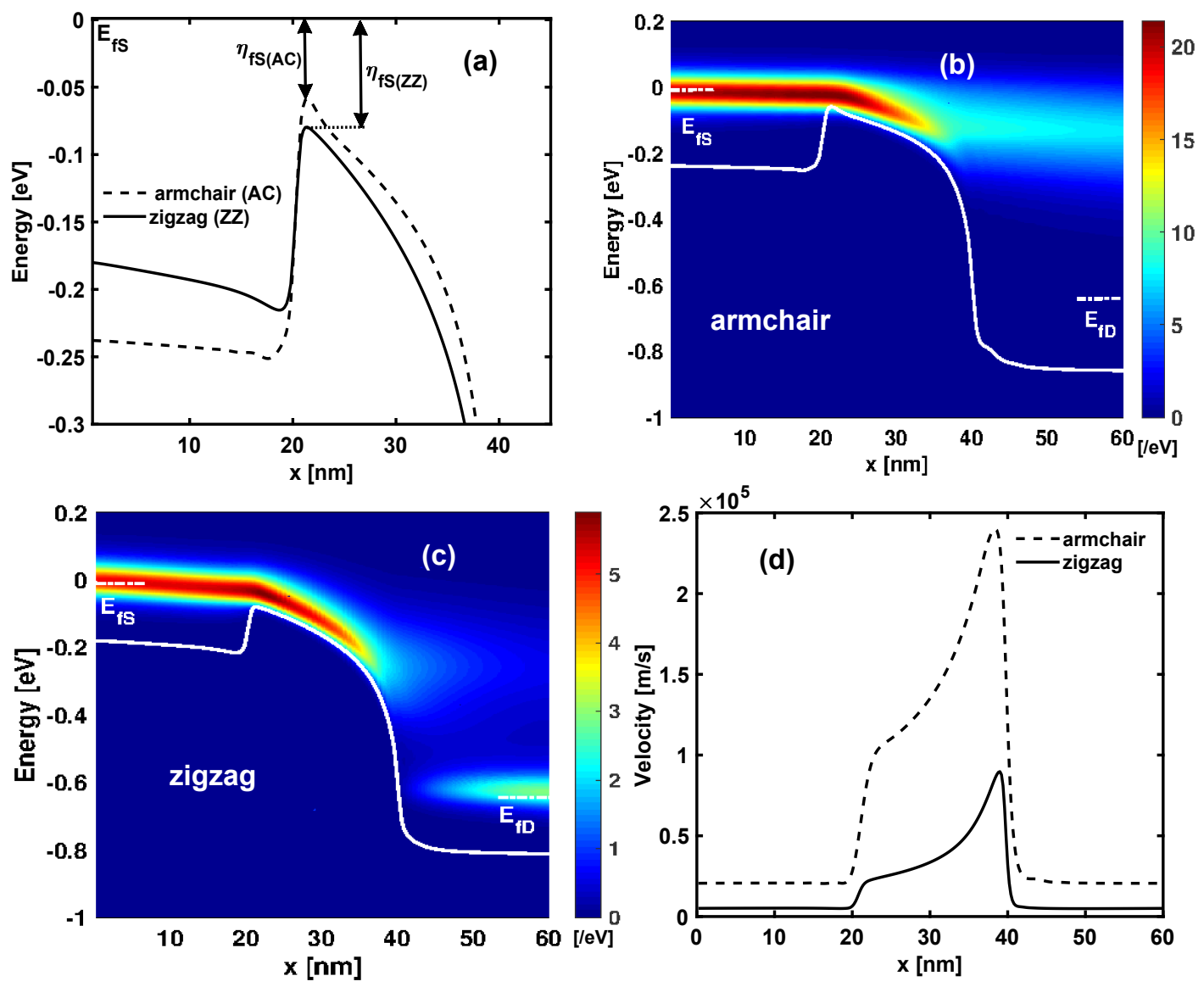

FIG. 5. (a) Potential barrier profile of n-MOSFET along AC and ZZ directions. It should be noted that the source potential profile bending at $\mathrm{ON}$ state arises due to the filling of states below the source contact by phonon emission which ultimately leads to better convergence [36]. $\eta_{\mathrm{fS}(\mathrm{ZZ}(\mathrm{AC}))}$ denotes the distance between the source Fermi level and top of the barrier along $\mathrm{ZZ}(\mathrm{AC})$. Since $\eta_{\mathrm{fS}(\mathrm{ZZ})}>\eta_{\mathrm{fS}(\mathrm{AC})}$, it results in increased backscattering and drain current loss along zigzag transport direction. Energy resolved current spectrum superimposed on the conduction band edge profile along (b) AC and along(c) ZZ directions. (d) Carrier drift velocity across the device length along both directions.

\section{RESULTS AND DISCUSSIONS}

We first discuss the effect of different phonon branches, both optical and acoustic, on the drain current of n- and p-MOSFETs. FIG. 4 (a)-(d), shows the dissipative and ballistic transfer characteristics of n- and p- type MOSFETs for AC and ZZ directions. In n-channel phosphorene MOSFETs, inelastic optical phonon scattering plays the most important role in degradation of ON state characteristics along both directions. FIG. 4(a) and (b) depict that ON state current is affected largely by the interaction between electron and optical phonon mode 7 in both n-AC and n-ZZ MOSFETs. The reason behind this phenomenon lies in the fact that optical phonon branch 7 has the highest deformation potential value and the lowest phonon energy (see TABLE. I), which leads to increased backscattering of carriers through both emission and absorption [38] of phonons. On the other hand, remaining optical modes with higher phonon energy and smaller deformation potential can only backscatter through emission, resulting in reduced current loss.

On the other hand, in p-channel MOSFETs, interaction between holes and optical phonon branches 9 and 11 strongly affect transport along both $\mathrm{AC}$ and $\mathrm{ZZ}$ as shown in FIG. 4(c) and (d). Indeed, phonon modes 9 and 11 have higher values of deformation potential than mode 7 in the valence band (see TABLE. I) and thus higher contribution in the total scattering rate of holes [30]. FIG. 4(c) and (d) also demonstrate some interesting phenomenon with respect to acoustic scattering in p-MOSFETs. From FIG. 4(d) it is found that effect of scattering between holes and acoustic phonon branch 3 is minimal on total drain current loss of p-ZZ MOSFET. However, the interaction between holes and acoustic phonon branch 2 results in severe degradation of drain current in p-AC MOSFET (see FIG. 4(c)). This phenomenon is attributed to the value of acoustic phonon deformation potential value along $\mathrm{AC}$ (i.e. hole-phonon 

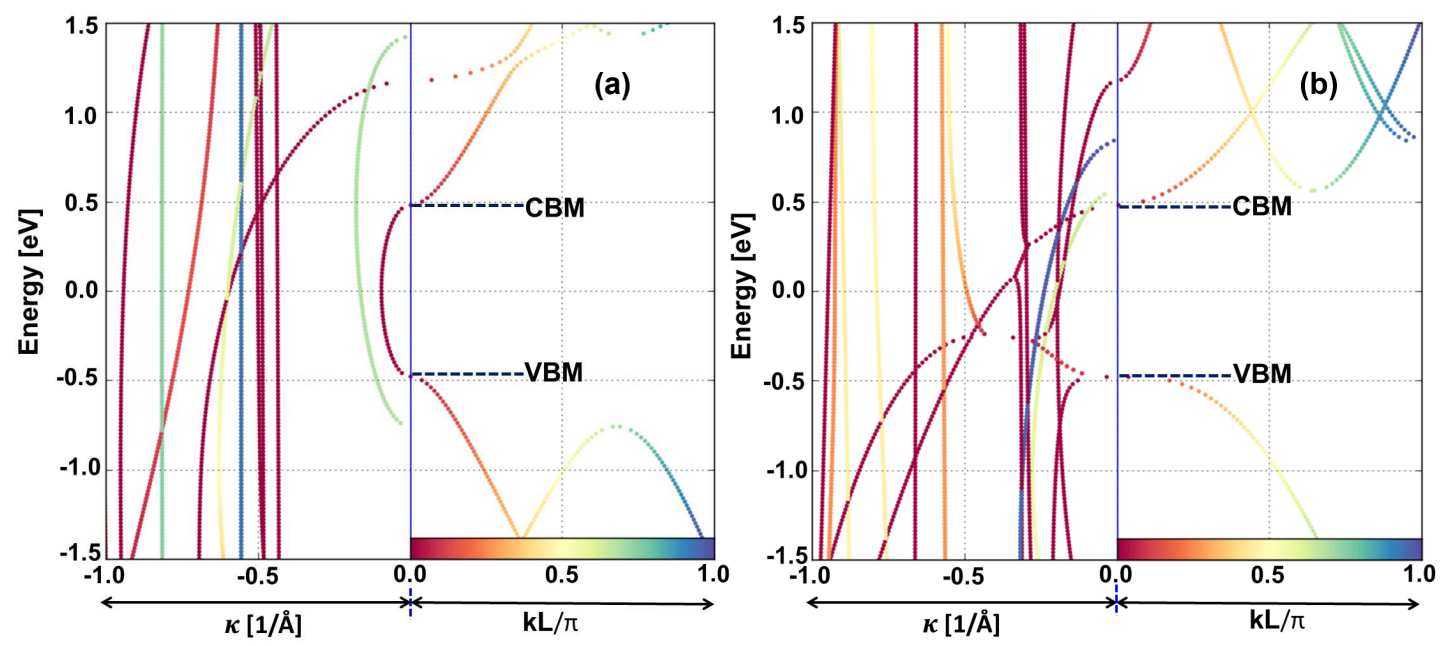

FIG. 6. Complex band structure along (a) [100] i.e. armchair and (b) [010] i.e. zigzag directions. The right-hand panel illustrates the real bands, where the solution $\mathrm{k}$ are normalized by the perpendicular layer separation L. The left hand panel portrays the complex bands against reciprocal Cartesian coordinates on the $\mathrm{x}$-axis. There is complete wrapping of imaginary bands between the conduction band minimum (CBM) and valence band maximum (VBM) along armchair direction. However, the imaginary bands along zigzag direction show multiple crossing between CBM and VBM.

mode 2) direction which is four times larger than the value along $\mathrm{ZZ}$ (i.e. hole-phonon mode 3 ) direction (see TABLE. I). It is further observed from FIG. 4(c) that contribution of acoustic phonon branch 2 becomes comparable to the one of optical branches 9 and 11 in p-AC MOSFET. This entails from comparable values of deformation potential of acoustic mode 2 and optical modes 9 and 11 in the valence band of phosphorene.

Nonetheless, all the devices n- and p- AC and ZZ, exhibit a decrease of $I_{\mathrm{D}}$ as $V_{\mathrm{G}}$ goes beyond the weak inversion region. At higher $V_{\mathrm{G}}$ the top of the barrier in both n- and p- MOSFETs along both directions, is pushed below the source Fermi level. This results in increased backscattering by optical phonons and significant loss of drain current $I_{\mathrm{D}}$ in the strong inversion region with respect to subthreshold region.

Additional insights on dissipative transport of phosphorene MOSFETS are provided in the following part of this section by comparing (i) same direction but different channel doping (i.e. $\mathrm{n}-\mathrm{AC}, \mathrm{p}-\mathrm{AC}$ and $\mathrm{n}-\mathrm{ZZ}, \mathrm{p}-\mathrm{ZZ}$ MOSFETs) and (ii) similar channel doping but different directions of transport (i.e. $\mathrm{n}-\mathrm{AC}, \mathrm{n}-\mathrm{ZZ}$ and $\mathrm{p}-\mathrm{AC}, \mathrm{p}-\mathrm{ZZ}$ MOSFETs).

Phosphorene MOSFETs along a given direction with different channel types i.e. $\mathrm{n}$ or $\mathrm{p}$, depict significant difference in ON-current (see FIG. 4(a)-(c) and (b)-(d)). This is reflected from the values of ballisticity of drain current which is calculated as: Ballisticity $\%=\left[I_{\mathrm{D}}\right.$ (dissipative $) / I_{\mathrm{D}}$ (ballistic $\left.)\right]$. Magnitude of ON-current is almost similar for n- and p-MOSFETs along AC direction $(2.34 \mathrm{~mA} / \mathrm{\mu m}$ and $2.46 \mathrm{~mA} / \mathrm{\mu m}$, respectively) in the ballistic regime due to comparable values of effective mass. However, the ballisticity for n-AC
MOSFET in the scattering regime is $42 \%$ whereas for $\mathrm{p}-\mathrm{AC}$ MOSFET is $63 \%$. This difference in dissipative ON state current arises due to higher values of deformation potential for optical phonon modes in the conduction band than in the valence band (see TABLE. I). The effective mass of holes is approximately 4.5 times larger than electrons along zigzag direction. As a consequence, ballistic ON-current in p-ZZ MOSFET is significantly less than n-ZZ MOSFET $(0.8 \mathrm{~mA} / \mu \mathrm{m}$ and $1.12 \mathrm{~mA} / \mu \mathrm{m}$, respectively). However, p-ZZ MOSFET surpasses n-ZZ MOSFET in terms of ballisticity by a significant amount ( $20 \%$ in $\mathrm{n}-\mathrm{ZZ}$ and $42.5 \%$ in $\mathrm{p}-\mathrm{ZZ}$ ). This results from sufficiently lower values of deformation potential along $\mathrm{ZZ}$ direction, of both acoustic and optical phonon modes in the valence band than in the conduction band (see TABLE. I). Overall n-type phosphorene MOSFETs undergo stronger scattering with respect to their $\mathrm{p}$ channel counterparts and hence demonstrate poor ballisticity.

Next, transfer characteristics in $\mathrm{n}$ and $\mathrm{p}$ channel MOSFETs along different directions are compared (see FIG. 4 (a)-(b) and (c)-(d)). These figures suggest that scattering starts to domninate the transfer characteristics in both $\mathrm{n}$ - and p-ZZ MOSFETs from lower values of gate voltage, $V_{\mathrm{G}}$. In other words, effect of scattering is stronger in case of phosphorene MOSFETs along zigzag direction. This phenomenon is explained with the help of FIG. 5 which shows conduction band profiles for both $\mathrm{AC}$ and $\mathrm{ZZ} \mathrm{n-}$ type devices. It should be noted that, though FIG. 5 shows only n-MOSFETs, similar argument applies for pMOSFETS. Along the ZZ direction, the transport effective mass is higher whereas the transverse effective mass is lower. Higher transport effective mass entails poor transmission and lower transverse effective mass leads 

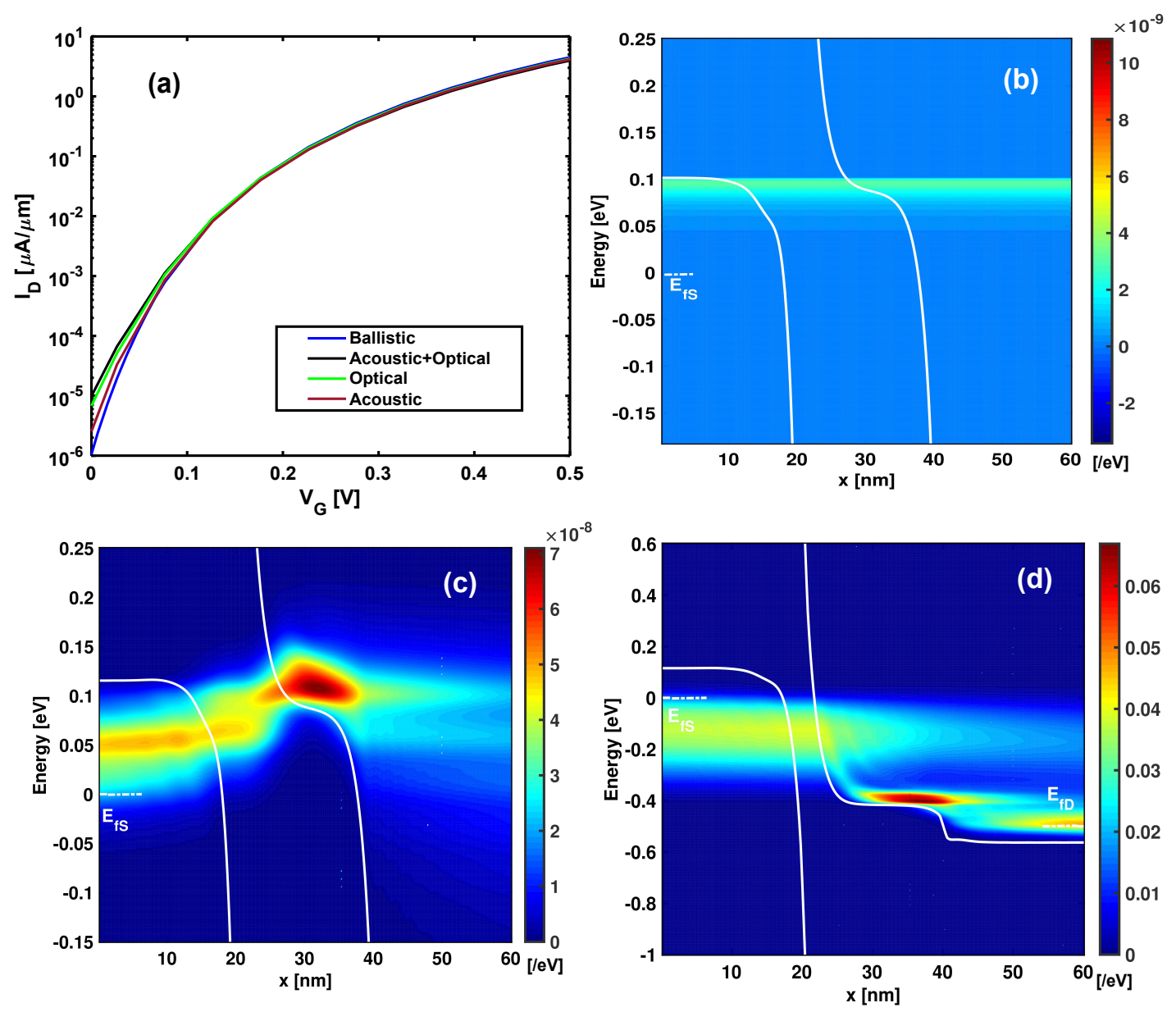

FIG. 7. (a) $I_{\mathrm{D}}-V_{\mathrm{G}}$ plot for p-i-n TFET. (b) Energy resolved current spectrum superimposed on the conduction band edge profile for phosphorene TFET along AC direction in the ballistic regime at OFF state (c) Same as (b) in the dissipative regime at OFF state and (d) Same as (c) in the dissipative regime at ON state. OFF state current in ballistic TFET yields lower values due to presence of higher tunneling barrier. However, in dissipative regime, OFF-current rises under the action of phonon absorption at the source-channel interface. On the other hand, in the ON state, negligible amount of carriers can backscatter into drain due to high potential barrier at the junction of channel and drain.

to smaller density of states. Both of these effects result in lower values of drain current $I_{\mathrm{D}}$. Therefore, $\mathrm{ZZ}$ MOSFETs require a smaller potential barrier in order to ensure the same $I_{\mathrm{D}}$ of AC MOSFETs. As a consequence, the difference betwen the source fermi level $\mathrm{E}_{\mathrm{fS}}$ and top of the barrier, $\eta_{\mathrm{fS}}$, is greater along $\mathrm{ZZ}$ direction (see FIG. 5(a)) and the effect of optical phonon backscattering becomes more important [39] in phosphorene ZZMOSFETs. Current spectrum plots in FIG. 5 (b) and (c) also show that inelastic emission process is stronger for ZZ MOSFET. This induces a decrease of the effective carrier velocity (calculated as $\mathrm{v}_{\mathrm{drift}}=\mathrm{I}_{\mathrm{D}} /(\mathrm{q} \times$ Charge $)$, $\mathrm{q}$ is electronic charge) with respect to $\mathrm{AC}$ device (see FIG. 5(d)), and subsequent space charge accumulation at the source end. It leads to significant modulation of the channel potential and enhances the scattering and current loss mechanism [38]. Therefore ZZ MOSFETs demonstrate poor performance under dissipative regime irrespective of the type of channel doping i.e. $\mathrm{n}$ or $\mathrm{p}$.
Aggressive scaling of channel lengths in MOSFET result in increase of OFF current in MOSFET as the subthreshold swing is limited by thermionic emission to 60 $\mathrm{mV} /$ dec. TFET is a gated $\mathrm{p}-\mathrm{i}-\mathrm{n}$ diode, which relies on the mechanism of BTBT for injection of carriers. This ensures the band pass filtering of high energy tails of source Fermi function which might result in low OFF current and sub-60 mV/dec subthreshold slope. The complex band structure of a material could provide useful guideline for its suitability as high performance TFET channel material. Wrapping of imaginary bands between conduction and valence band edges imply higher BTBT probability [40]. Estimate of BTBT probability under Wentzel-Kramers-Brillouin approximation, could be obtained from the area covered under the wrapped imaginary band (known as least action integral) which is usually small in case of low effective mass materials [40].

The complex band structure of phosphorene along [100] i.e. AC direction in FIG. 6(a) shows complete wrap- 
ping between conduction band and valence band edges. On the contrary, there are multiple crossings of imaginary bands between the conduction band minimum and valence band maximum in $\mathrm{ZZ}$ phosphorene as shown in FIG. 6(b). Some imaginary bands are close together with energy difference less than the energy of optical phonon $\hbar \omega_{\mathrm{ph}}$. This translates to strong phonon assisted tunneling (PAT) along ZZ direction at OFF state [41]. Treatment of multiple imaginary band crossings on TFET transfer characteristics is outside the scope of this paper since it should be calculated by considering a hamiltonian spanning more than two bands.

However transport calculation based on a two band $\mathbf{k} \cdot \mathbf{p}$ hamiltonian is sufficient for the cases where the imaginary band wraps itself between conduction and valance band edges. The transfer characteristics of phosphorene TFET along AC direction as obtained from selfconsistent transport calculations shows nominal effect of PAT throughout the gate bias window (see FIG. 7(a)) and dominance of optical phonon modes with respect to acoustic modes. FIG. 7(a) also shows that OFF current changes by one decade under PAT, with respect to ballistic transport. However, the change in subthreshold swing spans over a very small region of gate voltage (approximately $0.1 \mathrm{~V}$ ) and the dissipative $I_{\mathrm{D}}$ quickly catches up with the ballistic $I_{\mathrm{D}}$. At low gate bias band bending at source channel interface is not severe enough to facilitate direct tunneling in contrast to ballistic case (see FIG. 7(b)). However, OFF current rises because transmission of carriers mostly occurs by phonon absorption at the source side which thermalizes on reaching the drain contact $[41,42]$ as depicted in FIG. 7(c). However, at high gate bias, the effect of PAT diminishes. Conduction band in the channel is pushed well below the valence band in the source and transmission is dominated by direct BTBT as shown in FIG. 7(d). The optical phonons that are emitted at the drain region are not able to backscatter into the source due to the presence of large potential barrier at the channel-drain junction. As a result there is negligible current loss at ON state, which is just opposite of MOSFET.

\section{CONCLUSION}

The effect of different types of electron phonon coupling on the transfer characteristics of phosphorene nand p-MOSFETs along both $\mathrm{AC}$ and $\mathrm{ZZ}$ directions are investigated through self consistent NEGF-SCBA equations. Results suggest that zero order optical phonon mode having the highest deformation potential constant and the lowest energy, bears the strongest effect on degradation of ON-current by scattering in all the devices except p-AC MOSFET. Effect of carrier-phonon scattering is found to be more pronounced in transport along $\mathrm{ZZ}$ direction than armchair direction for MOSFETS of similar channel doping. However, p-MOSFETs outperform n-MOSFETs in terms of ballisticity along a specific direction of carrier transport. The complex band structure of phosphorene provides an interesting insight. It suggests that BTBT probability along ZZ direction would be hugely compromised due to multiple imaginary band crossing inside the band gap. However, effect of scattering on PAT is found to be minimal for AC TFET in terms of subthreshold swing.

\section{ACKNOWLEDGMENTS}

This work was supported in part by the Science and Engineering Research Board, Department of Science and Technology, Government of India, under Grant SB/S3/EECE/0209/2015, in part by the Robert Bosch Cyber Physical System, Indian Institute of Science Bangalore, under Grant RBC00011, and in part by the Raman-Charpak Fellowship, Centre Franco-Indien pour la Promotion de la Recherche Avancée under Grant RCFIN-133.
[1] B. Radisavljevic, M. B. Whitwick, and A. Kis, ACS nano 5, 9934 (2011).

[2] S. B. Desai, S. R. Madhvapathy, A. B. Sachid, J. P. Llinas, Q. Wang, G. H. Ahn, G. Pitner, M. J. Kim, J. Bokor, C. Hu, et al., Science 354, 99 (2016).

[3] H. Fang, S. Chuang, T. C. Chang, K. Takei, T. Takahashi, and A. Javey, Nano letters 12, 3788 (2012).

[4] L. Li, Y. Yu, G. J. Ye, Q. Ge, X. Ou, H. Wu, D. Feng, X. H. Chen, and Y. Zhang, Nature nanotechnology 9, 372 (2014).

[5] H. Liu, A. T. Neal, Z. Zhu, Z. Luo, X. Xu, D. Tománek, and P. D. Ye, ACS nano 8, 4033 (2014).

[6] K. Novoselov, D. Jiang, F. Schedin, T. Booth, V. Khotkevich, S. Morozov, and A. Geim, Proceedings of the National Academy of Sciences 102, 10451 (2005).
[7] W. Lu, H. Nan, J. Hong, Y. Chen, C. Zhu, Z. Liang, X. Ma, Z. Ni, C. Jin, and Z. Zhang, Nano Research 7, $853(2014)$.

[8] Z. Guo, H. Zhang, S. Lu, Z. Wang, S. Tang, J. Shao, Z. Sun, H. Xie, H. Wang, X.-F. Yu, et al., Advanced Functional Materials 25, 6996 (2015).

[9] L. Kou, C. Chen, and S. C. Smith, The journal of physical chemistry letters 6, 2794 (2015).

[10] X. Luo, Y. Rahbarihagh, J. C. Hwang, H. Liu, Y. Du, and D. Y. Peide, IEEE Electron Device Letters 35, 1314 (2014).

[11] N. Haratipour, S. Namgung, S.-H. Oh, and S. J. Koester, ACS nano 10, 3791 (2016).

[12] X. Cao and J. Guo, IEEE Transactions on Electron Devices 62, 659 (2015). 
[13] H. Ilatikhameneh, T. Ameen, B. Novakovic, Y. Tan, G. Klimeck, and R. Rahman, Scientific reports 6, 31501 (2016).

[14] A. Szabo, R. Rhyner, H. Carrillo-Nunez, and M. Luisier, in Electron Devices Meeting (IEDM), 2015 IEEE International (IEEE, 2015) pp. 12-1.

[15] M. Luisier, A. Szabo, C. Stieger, C. Klinkert, S. Brück, A. Jain, and L. Novotny, in Electron Devices Meeting (IEDM), 2016 IEEE International (IEEE, 2016) pp. 54.

[16] M. Brahma, A. Kabiraj, and S. Mahapatra, in VLSI Design and 2019 18th International Conference on Embedded Systems (VLSID), 2019 32nd International Conference on (IEEE, 2019).

[17] "International Technology Roadmap for Semiconductors," http://www.itrs2.net/2013-itrs.html, last accessed: 2017-11-26.

[18] G. Kresse, Phys. Rev. B 54, 169 (1996).

[19] G. Kresse and J. Furthmüller, Physical review B 54, 11169 (1996).

[20] G. Kresse and J. Furthmüller, Computational materials science 6, 15 (1996).

[21] G. Kresse and D. Joubert, Physical Review B 59, 1758 (1999).

[22] J. P. Perdew, K. Burke, and M. Ernzerhof, Physical review letters $\mathbf{7 7}, 3865$ (1996).

[23] J. Heyd, G. E. Scuseria, and M. Ernzerhof, The Journal of chemical physics 118, 8207 (2003).

[24] J. Qiao, X. Kong, Z.-X. Hu, F. Yang, and W. Ji, Nature communications 5, 4475 (2014).

[25] A. Rodin, A. Carvalho, and A. C. Neto, Physical review letters 112, 176801 (2014).

[26] A. Togo and I. Tanaka, Scripta Materialia 108, 1 (2015).

[27] "QuantumWise Atomistix ToolKit (ATK) with Virtual NanoLab," http://quantumwise.com/, last accessed: 2017-11-26.

[28] D. Hamann, Physical Review B 88, 085117 (2013).
[29] M. Schlipf and F. Gygi, Computer Physics Communications 196, 36 (2015).

[30] M. Elahi and M. Pourfath, Journal of Physics: Condensed Matter 30, 225701 (2018).

[31] K. Kaasbjerg, K. S. Thygesen, and K. W. Jacobsen, Physical Review B 85, 115317 (2012).

[32] S. Datta, Quantum transport: atom to transistor (Cambridge university press, 2005).

[33] M. Anantram, M. S. Lundstrom, and D. E. Nikonov, Proceedings of the IEEE 96, 1511 (2008).

[34] M. Bescond, D. Logoteta, F. Michelini, N. Cavassilas, T. Yan, A. Yangui, M. Lannoo, and K. Hirakawa, Journal of Physics: Condensed Matter 30, 064005 (2018).

[35] U. Aeberhard and R. Morf, Physical Review B 77, 125343 (2008).

[36] M. Luisier and G. Klimeck, Physical Review B 80, 155430 (2009).

[] A. Svizhenko and M. Anantram, IEEE Transactions on Electron Devices 50, 1459 (2003).

[] S. Jin, Y. J. Park, and H. S. Min, Journal of Applied Physics 99, 123719 (2006).

[] Y. Lee, M. Bescond, D. Logoteta, N. Cavassilas, M. Lannoo, and M. Luisier, Physical Review B 97, 205447 (2018).

[37] R. Lake, G. Klimeck, R. C. Bowen, and D. Jovanovic, Journal of Applied Physics 81, 7845 (1997).

[38] H. Tsuchiya and S.-i. Takagi, IEEE Transactions on Electron Devices 55, 2397 (2008).

[39] S. O. Koswatta, S. Hasan, M. S. Lundstrom, M. Anantram, and D. E. Nikonov, IEEE Transactions on Electron Devices 54, 2339 (2007).

[40] R. K. Ghosh and S. Mahapatra, IEEE Journal of the electron devices society 1, 175 (2013).

[41] M. Luisier and G. Klimeck, Journal of Applied Physics 107, 084507 (2010).

[42] S. O. Koswatta, M. S. Lundstrom, and D. E. Nikonov, Applied Physics Letters 92, 043125 (2008). 